\title{
EXPERIENCIA EN LA TRANSPLANTACIÓN DE LA CLASE MÁGICA AL ENTORNO MEXICANO
}

\section{EXPERIENCE IN TRANSPLANTING LA CLASE MAGICA TO THE MEXICAN ENVIROMENT}

\section{Dra. Verónica García Martínez}

\section{Dra. Silvia Patricia Aquino Zúñiga \\ Jessica del Carmen López Laynez}

\begin{abstract}
Resumen
Se describe una experiencia de intervención educativa de estudiantes de pregrado en escuelas primarias inspirado en La Clase Mágica (LCM), modelo construido principalmente sobre las bases teóricas del socio constructivismo de Vigotsky. LCM se originó con el propósito de ayudar a niños migrantes latinos a adaptarse a la cultura norteamericana, posteriormente su aplicación evolucionó a diversos entornos. En México, se instrumentó a través de un proyecto que buscaba que estudiantes de servicio social y prácticas profesionales de la Licenciatura en Ciencias de la Educación, apoyaran a niños de primaria en la asignatura de español.
\end{abstract}


La implementación inició en febrero de 2016 y continuó hasta junio de 2017. Este trabajo da cuenta del proceso de trasplantación del modelo al entorno mexicano. Los resultados indican que la institución, niños, estudiantes universitarios, y padres de familia involucrados se benefician a pesar de algunas debilidades del modelo relacionadas al contexto cultural y del entorno.

\title{
Palabras Clave
}

Trasplantación, la Clase Mágica, modelo de intervención educativa, socio constructivismo.

\begin{abstract}
The aim of this paper is to describe an experience of educational intervention based on La Clase Magica (LCM) and conducted by undergraduate students in a public elementary school. LCM is a curriculum model based on Vigostsky's construtivism theory. The purpose of LCM is to help Latino migrant children to adapt into the American culture; the application of this model has evolved to different environments. In Mexico, the model was implemented through a research project where undergraduate students during their social service and professional practices of the Degree in Educational Science support elementary students to improve their skills in the Spanish subject. The implementation lasted from February 2016 to June 2017. This work describes the process of transplanting the model to the Mexican environment. The results indicate that the institution, children, parents and undergraduate students benefited despite some weaknesses of the model related to the context and cultural environment.
\end{abstract}

\section{Key Words}

Transplantation, educative intervention mode, socio constructivism.

\section{Antecedentes}

En febrero de 2016, comenzó una proceso de intervención educativa en dos escuelas primarias de Tabasco, México, basado en La Clase Mágica (LCM), un modelo de intervención educativa iniciado en la Universidad de California hace 25 años, con el propósito de ayudar a usar la tecnología a los niños de minorías hispanas en Estados 
Unidos de América (Vásquez, 2003). El modelo tiene su fundamento teórico en la pedagogía de la pregunta de $\mathrm{P}$. Freire, el método dialéctico, el socio-constructivismo, la noción de cultura y la acción lúdica de Vigotsky; la teoría de los capitales de Bourdieu, y la Quinta Dimensión de Cole. Este programa se amplió a otros propósitos formativos dada su plasticidad, lo que permitió su adaptación en otros Estados de la Unión Americana y en países como España, Alemania, Argentina y recientemente Colombia, con propósitos específicos y de acuerdo a las necesidades en cada caso.

Una característica del modelo es el papel que juegan los estudiantes de pregrado que fungen como asistentes con los niños, y de las familias participantes en el programa. El impacto de aplicación de LCM ha sido probado a través de dos décadas en generaciones de niños, estudiantes y familias, que fueron beneficiados con el programa. Estudios longitudinales realizados en California, revelaron que el $90 \%$ de los niños que participaron tres o más años en LCM se inscribieron o habían terminado incluso un grado en estudio superior, lo cual no es habitual en la comunidad latina. El $32 \%$ de los estudiantes de secundaria que apoyaron como asistentes, planeaban obtener su grado profesional, 20 de los estudiantes universitarios participantes indicaron que su exposición a LCM les permitió proseguir estudios de posgrado y siete de ellos se convirtieron en miembros de la facultad (Bustos, Vásquez y Riojas, 2014). Los resultados además destacaron el crecimiento moral e intelectual de los estudiantes que participaron en él, de los padres y de otras personas que de una u otra manera se involucraron en su desarrollo (Martínez, 2012; Martínez, 2014).

LCM se ha aplicado con diversos fines. En Texas, se enfocó en un inicio a los estudiantes de comunidades latinas con bajo desempeño escolar en el idioma inglés, matemáticas y ciencias (Willey, López, Torres y Licon, 2014). Este proyecto fue coordinado por la universidad estatal con apoyo de otras organizaciones, con la finalidad de disminuir la inequidad de oportunidades de inserción social, y logró resultados que impactaron a los padres de familia (Claeys y Muñoz, 2014). En el modelo texano se implementaron otras innovaciones orientadas hacia la utilización de la tecnología para la adquisición de competencias (Sánchez, Yuen, Shonle, De Hoyos, Santillán y García, 2014; Alanís, 2014) y la construcción de identidades (Ek, García, y Garza, 2014). Los resultados obtenidos fueron tan sorprendentes que fue tema de discusión a nivel estatal sobre política educativa (López y Valenzuela, 2014).

En España, la adaptación de LCM sirvió para atenuar inequidades sociales con 
los grupos de gitanas del sur del país (Macías-Gómez y Vásquez, 2014). Coordinado por la Universidad de Pablo Olavide en Sevilla y con apoyo de organizaciones, el programa ofreció a las mujeres marginadas recursos para enfrentar las nuevas realidades sociales y técnológicas, empoderando a un grupo tradicionalmente vulnerable que se negaba a dejar su herencia cultural (Gómez y Vásquez, 2014). En el caso de Colombia, el programa buscó habilitar a los niños de nivel básico en el idioma inglés y sensibilizarlos en el cuidado ecológico.

Como puede advertirse, este modelo de intervención educativa puede enfocarse tanto a niños como a adultos. El modelo se implementa a través de una universidad, la cual coordina y provee los recursos humanos, (que son estudiantes y profesores) y a veces también los materiales para su puesta su en marcha. Ya sea a través de alguna asignatura relacionada con la educación o por medio de otra estrategia de agrupamiento, se habilitan y supervisan a jóvenes de los últimos grados para que sirvan como facilitadores de las actividades estructuradas (lúdicas) que estimulan en el grupo la participación, la colaboración y la adquisición de competencias en asignaturas específicas.

Los estudiantes universitarios realizan además de las actividades de intervención pedagógica labores de investigación y recogida de datos sobre las experiencias y evolución de los participantes. Debe haber detrás de toda esta intervención una estrategia organizada que comprenda la dotación de bagaje teórico y procedimental necesario para el cabal cumplimiento de las metas. La capacitación es de carácter disciplinar, metodológico, teórico y práctico. Es ahí donde intervienen profesores e investigadores que les proveen del entramado que les permita desarrollar sus competencias para hacer frente a la intervención. De modo que en este modelo (figura 1) participan universidad y unidad receptora a través de sus principales actores (estudiantes, profesores e investigadores y directivos, profesores, alumnos y padres de familia, respectivamente). 


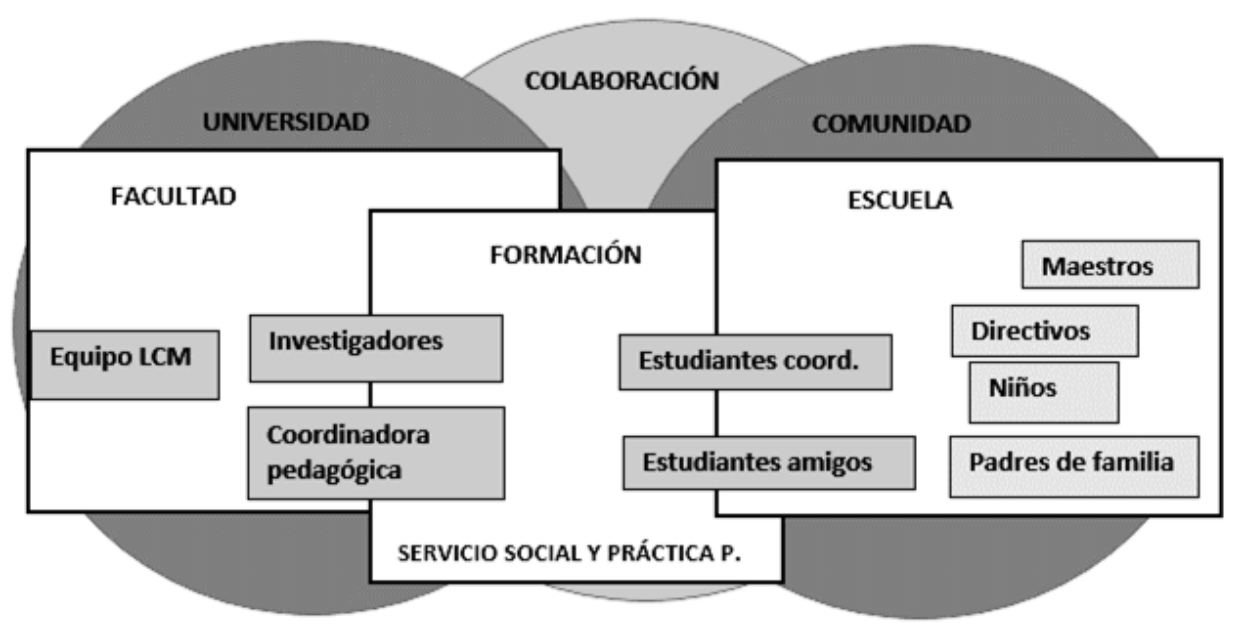

Figura 1. Participantes en el modelo de intervención LCM. Fuente: Elaboración propia.

La unidad emisora y receptora en este modelo no cambian: la universidad (unidad emisora) y un grupo que requiera de una intervención educativa que les permita mejorar sus condiciones de vida en un determinado contexto (unidad receptora). La esencia del modelo radica en la intervención sostenida que realizan los universitarios en los grupos necesitados de apoyo. Los estudiantes se sustituyen cada cierto tiempo de acuerdo a los ciclos escolares, pero el propósito se mantiene, así como la coordinación del mismo, en ello radica su sostenibilidad. Esto ha sido posible en diferentes escenarios gracias al proceso de trasplantación del modelo.

En la LCM prevalecen las situaciones didáctico- lúdicas, sea cual fuere el fin que se proponga en una intervención, y hay elementos que integran el modelo aunque no siempre se utilicen: el elemento humano constituido por los amigos, y el material por los artefactos: el laberinto, El Maga, y las task card (tarjetas de tarea), son algunos empleados para facilitar las actividades de aprendizaje dentro de los sitios. Para Vásquez (2003) este sistema genera una metodología en acción que se reconstruye de acuerdo a los propósitos que se establezcan en cada contexto.

Los amigos son los estudiantes que realizan la intervención y desarrollan una relación empática con los beneficiarios. El laberinto con apoyo de las tarjetas de tarea, representan un juego que va subiendo en complejidad de acuerdo la madurez de los sujetos. El Maga (The wizard), es un personaje imaginario con el que los niños interactúan y que corrobora con otros artefactos en la Quinta Dimensión, una estructura 
no jerárquica en las relaciones sociales del sitio (Vásquez, 2003, p. 158). Este conjunto de artefactos (como se denominan en el modelo) tienen presencia en la trasplantación aunque su uso depende de los propósitos de la intervención.

\subsection{Trasplantación de La Clase Mágica}

Trasplantar La Clase Mágica es una metáfora, significa "sembrar" el modelo en otros contextos. Vásquez (2003), autora del modelo, explica que éste se enfoca en los trabajos tempranos de los primeros años del proyecto iniciados en E.U, pero también tiene una presencia en el ámbito nacional e internacional, en virtud que se ha sembrado o "trasplantado" a otras comunidades culturales. Es así como surge la idea de hacer la analogía entre La Clase Mágica y una planta.

La experiencia de trasplantación de LCM trae consigo una serie de transformaciones y adaptaciones y que es necesario tomarle importancia al nuevo contexto "a la nueva tierra" que la adaptación al nuevo medio implica el establecimiento de nuevos objetivos, la adaptación a culturas y a costumbres diferentes, a una organización político cultural distinta (Vásquez, 2003; Vásquez y Marcello, 2009; Bustos et al., 2014; Santos, 2016). En cuanto a los cuidados de esta "planta" es necesario elaborar un nuevo plan de educación, establecer y reformular las capacitaciones del personal, rediseñar las técnicas y los materiales para las actividades etc. La supervivencia del modelo en el nuevo contexto dependerá en gran parte del interés y de la disposición del sembrador (universidad) y de la tierra nueva (la comunidad)

El proyecto Shere Rom en España aborda la experiencia de trasplantación en minorías de niños y adolescentes gitanos (Crespo, Lalueza, Lamas, Padrós y Sànchez, 2014). Crespo et al. (2014) consideran que no se trata simplemente de trasladar o implantar el modelo LCM en la escuela; el resultado es un tipo de actividad diferente y nueva es un "tercer objeto", en términos de Egeström (2007, citado por Crespo, 2014), fruto de la contraposición del modelo LCM con el modelo clásico escolar.

La trasplantación tiene que ver con una capacidad de adaptabilidad y apropiación del modelo en el nuevo contexto, para Rogoff, (1993) significa una especial sensibilidad a las características y necesidades de los contextos institucionales en los que se realizará, el proyecto debe adaptarse a la cultura local. Si dentro de las escuelas no hay un currículo cerrado de actividades, el diseño concreto de éstas debe atender a 
las características del grupo, los intereses de los maestros, las necesidades educativas de los niños y la dinámica de las escuelas.

Otro aspecto esencial que debe considerarse es el contexto social, las características del barrio y los marcos culturales comunitarios al que pertenecen las familias de los alumnos, por tanto la trasplantación de LCM a una escuela debe suponer la explícita consideración de las culturas que convergen en la organización educativa o grupo a apoyar. Esta adaptabilidad y apropiación tienen un estrecho vínculo con la sostenibilidad del proyecto a mediano y largo plazo. Es importante conseguir que en el proceso de trasplantación los agentes locales se apropien del proyecto y logren desarrollarlo de manera autónoma.

El establecimiento de metas compartidas entre universidad y comunidad contribuye de manera significativa en el éxito de la trasplantación; sin embargo, esto no quiere decir que ésta sea un proceso fácil puesto que todos los participantes suelen poseer lenguajes sociales distintos, y diferentes valores y formas de interpretar la vida (Crespo et al., 2014) Esto supone que la trasplantación en la nueva tierra trae consigo obstáculos, pero no es imposible obtener resultados positivos.

En el caso de México, la trasplantación fue posible gracias a un proyecto de investigación financiado por el fondo UC Mexus-CONACYT de la Universidad de California (UC) y el Gobierno Mexicano, coordinado por Olga Vásquez, creadora del modelo y Verónica García, investigadora de la Juárez Autónoma de Tabasco (UJAT). El proyecto tuvo lugar en dos escuelas de un municipio del estado de Tabasco, las cuales fueron elegidas porque se encontraban en áreas cuyas poblaciones son flotantes y hasta cierto punto marginales. En este sentido, uno de los principios de LCM es atender a las poblaciones vulnerables: los niños migrantes, mujeres gitanas, estudiantes rurales, son algunos de los grupos a los que se ha enfocado este modelo. La particularidad del mismo es su adaptabilidad al medio, sin embargo, hay que comprender con antelación su complejidad. A continuación se relata el proceso de trasplantación en el contexto referido.

\section{Descripción de la intervención: objetivos, metodología y resultados.}

Los objetivos. En el entorno mexicano, LCM fue implementada por la UJAT en el estado de Tabasco, ubicado en el sureste de México. Fueron varios los objetivos 
establecidos en este proyecto: a) vincular a la Universidad con el sub sistema de educación básica, b) ofrecer un espacio en el contexto real de práctica profesional para estudiantes universitarios de formación educativa y c) ayudar a los niños de escuelas primarias a incrementar sus habilidades en Español, dado que es una de las asignaturas en donde el nivel de logro es deficiente. En el Programa Sectorial de Educación (PSE) 2013-2018 se mencionan el desfavorable resultado que ha tenido el país en la prueba PISA (ubicado en el 53 de 65 países) sobre todo en matemática y español.

La idea para la aplicación parte de lo enunciado en el PSE, que considera que "la comunidad escolar está desprovista de apoyos, mecanismos, sistemas y recursos que le faciliten su labor educativa" (p. 24), además advierte que la escuela pública debe ser la promotora del cambio y la transformación social y recomienda revisar el modelo educativo pues los materiales no han tenido la diversidad deseable. En este sentido se consideró a LCM como una oportunidad de contribuir al mejoramiento de la calidad de la educación en el nivel básico. Aparte de atender a minorías y grupos vulnerables, el modelo persigue fines investigativos. En este sentido y al tener una base constructivista, los universitarios deben registrar sus experiencias como parte del proceso, para ser después consideradas como insumo en el reporte de resultados. Sigue un método de corte cualitativo o interpretativo, lo cual se resumen a continuación.

La metodología. Este proyecto responde al esquema de intervención y se hizo un seguimiento de tipo cualitativo. Se aplicó en dos escuelas primarias durante tres semestres, en quinto y sexto grado con un número promedio de 40 alumnos por año. Se solicitó el permiso de las autoridades educativas, del director de la escuela y de las maestras encargadas de los grupos para que se iniciara el proyecto de intervención con un grupo de estudiantes universitarios que en el modelo se denominan como "amigos".

Los estudiantes de la Universidad estatal que realizaron la intervención fueron jóvenes de la Licenciatura en Ciencias de la Educación, en promedio 16 por grupo en cada semestre. El primero de ellos trabajó de febrero a junio de 2016 (en julio es el verano y las vacaciones en las escuelas), el segundo de agosto a diciembre de 2016, y el tercero de febrero a junio de 2017. Fue durante un año y medio que jóvenes universitarios visitaron las escuelas primarias donde realizaron actividades apegadas al 
currículum en la asignatura de Español, con énfasis en comprensión lectora a solicitud de las profesoras encargadas de los grupos. A decir de ellas ésta representa una necesidad imperante entre los pequeños. El propósito fue incrementar las habilidades en esta materia entre los niños de quinto y sexto grado.

Para llevar a efecto la intervención en las escuelas, al interior de la universidad se reclutaron a jóvenes que quisieran realizaf su servicio social o prácticas profesionales en este programa. Estos jóvenes recibieron capacitación de profesores especialistas en diferentes aspectos que comprende la misma, principalmente cuatro: a) conocimiento de LCM, b) estrategias didácticas, c) modelos pedagógicos y d) técnicas de investigación. Con apoyo de una asesora pedagógica, realizaron la planeación de las actividades de aprendizaje lúdicas que desarrollarían con los niños a lo largo de la intervención. Durante este tiempo cada "amigo" atendió de cinco a ocho niños (Figura 2).

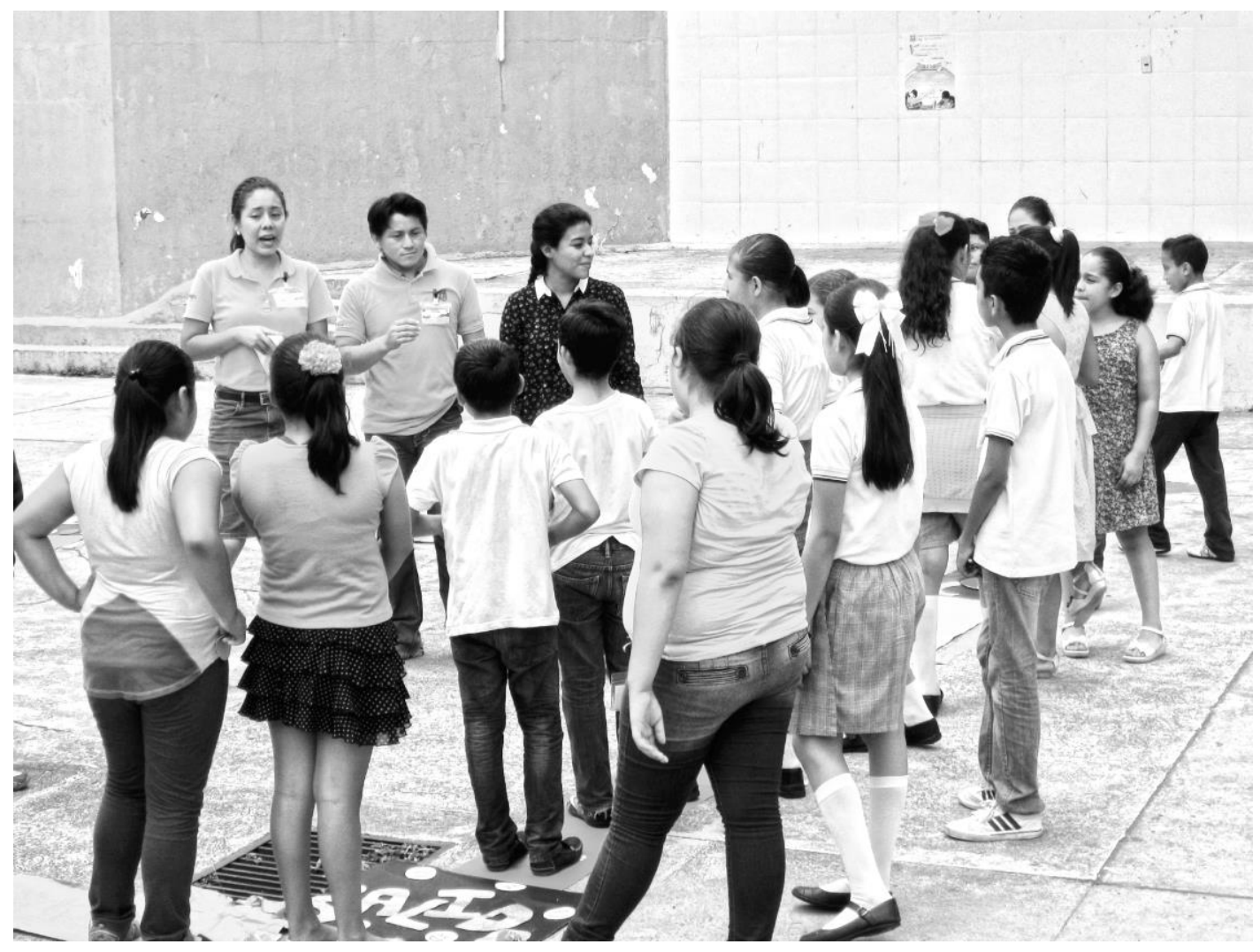

Figura 2. Amigos y niños participantes 
La metodología de investigación de corte interpretativo, se desarrolló a lo largo de la intervención. Por medio principalmente de dos técnicas, observación y entrevistas, se logró el acopio de la información que permitió alcanzar uno de los objetivos intrínsecos trazados que era la reconstrucción de la implementación. En apoyo a este acopio hubo un grupo de estudiantes observadores que recogieron las experiencias de trasplantación del modelo en este entorno a través de formatos de registro. Las entrevistas también sirvieron para tal fin así como las notas de campo de los propios participantes. Todo esto permitió la reconstrucción del proceso de trasplantación de LCM como parte de los resultados del proyecto, lo cual se detalla en la siguiente sección.

\subsection{Etapas de LCM en la trasplantación al entorno mexicano}

En el proceso de trasplantación de LCM en Tabasco se identificaron cuatro fases: 1) diagnóstico y negociación, 2) organización y planeación, 3) capacitación y 4) diseño e implementación. A continuación se describe cada una de ellas.

\subsubsection{Fase de diagnóstico y negociación}

Esta fase consistió en la vinculación entre la Universidad a través de los responsables del proyecto y las autoridades educativas de la entidad, para lo cual se realizaron reuniones de trabajo con los directores de las primarias. Fueron seleccionados los sitios con un perfil definido de organizaciones educativas que fueran escuelas de horario extendido, a fin de disponer de tiempo para realizar la intervención. LCM opera en horarios extracurricular, generalmente fuera de las instalaciones de las escuelas y con la autorización de los padres de los niños, lo cual en México resulta muy complicado. En este sentido se optó por aprovechar la condición del horario extendido y la infraestructura de la escuela.

Otra característica considerada fue que atendieran a poblaciones marginadas por su situación social y económica. Se asignaron dos escuelas para trabajar en un horario de 13:00 a 14:30 horas, con el previo consentimiento de las profesoras que estaban al frente de los grupos de cuarto y quinto grados', quienes a su vez avisaron a los padres de familia de la intervención. 


\subsubsection{Fase de organización y planeación}

Para esta fase fue determinante ajustar los tiempos del calendario escolar de educación básica con el de los universitarios, ya que de este emparejamiento dependía la organización de las actividades. La primera fue el desarrollo de un diagnóstico de la población escolar que sería intervenida, lo cual se efectuó mediante entrevistas y formularios que se llenaron con los datos de los niños. Posteriormente se les aplicó una evaluación sobre temas relacionados con contenidos de español de acuerdo a la currícula de cada grado. Los resultados de ambos diagnósticos arrojaron que la población de niños de esas escuelas pertenece a familias trabajadoras y muchas veces mono parentales, y poseen similares resultados con respecto al nivel de conocimientos en la asignatura.

En esta misma etapa se terminó de reclutar al grupo de participantes universitarios que serían los amigos de esos niños durante diez semanas. Estos estudiantes fueron convocados por medio del servicio social y las prácticas profesionales, que son dos actividades obligatorias en su trayectoria escolar. También se consolidó el equipo de profesores que apoyarían directamente la intervención: una responsable, una psicóloga pedagoga, una especialista en didáctica, un antropólogo y dos profesores de la Licenciatura en Ciencias de la Educación.

Los estudiantes realizaron las primeras entradas a las escuelas para "romper el hielo" e irse ubicando en el colectivo como parte del mismo. No fue sencillo, para jóvenes con poca experiencia en la pedagogía práctica, pero con un bagaje teórico importante que les permitió solventar los obstáculos. Los universitarios acordaron llegar dos veces por semana a cada escuela y los viernes planear las actividades de la siguiente. Los inicios de la intervención concordaron con la capacitación complementaria por parte del equipo tanto de la UJAT como de la Universidad de California.

\subsubsection{Fase de capacitación}

Por ser un modelo de innovación en el contexto mexicano, fue menester que profesores y estudiantes de la UC ofrecieran algunos cursos orientadores del modelo al equipo de la UJAT, por lo que realizaron varias visitas desde el inicio y durante los tres semestres de las intervenciones. Esta capacitación se engarzó con la de orientación 
pedagógica y de micro enseñanza, en virtud de que las prácticas que desarrollan los estudiantes en la intervención tienen una fuerte base socio constructivista, pero incorporan elementos de otros enfoques que inciden necesariamente en el practicum de los jóvenes participantes.

Aunado a la anterior, se les formó en técnicas de investigación cualitativa, dado que su papel no solo se remite a la acción, sino también a la investigación. En este sentido, un antropólogo les habilitó para la observación y el registro de datos así como para el uso del diario de campo. Los estudiantes al término de cada sesión debían llenar unas hojas de registro con sus comentarios, que iban desde la perspectiva macro a la micro, en torno a su experiencia diaria. Uno de los objetivos es fue hacerlos reflexionar sobre su propia práctica. También se les dio un taller de competencias conversacionales, que les fue impartido por una estudiante de la Maestría en Innovación e Intervención de la Práctica Educativa participante en el proyecto.

Con toda esta "artillería" los universitarios avanzaban integrando toda la complejidad de un modelo que se ponía recientemente en práctica, mismo que fomenta competencias en los principales participantes del mismo, dada la alta interacción que demanda. La construcción de esta armadura, permitió la implementación de LCM en un nuevo entorno, que derivó en una trasplantación, con sus propias condiciones.

\subsubsection{Fase de diseño e implementación}

Esta fue la cuarta y última fase en la cual una de las profesoras participantes les orientó sobre las teorías pedagógicas que sustentan el trabajo educativo, y otra sobre didáctica y micro enseñanza. Sobre esta base se llevó a cabo una planeación de actividades preponderantemente lúdicas (cuarta etapa), pero con un propósito didáctico y de reforzamiento de competencias en español.

Los estudiantes junto con las asesoras adaptaron los elementos didácticos de LCMii para atender a los objetivos establecidos respecto a las habilidades de español. La idea central fue plasmar la propuesta del modelo a través de actividades de aprendizaje sustentadas en la teoría constructivista, ajustadas al concepto de zona de desarrollo próximo establecido por Vygotsky. Los estudiantes elaboraron sus propias propuestas didácticas para la intervención.

De este modo se elaboraron dos grandes proyectos, uno para quinto grado, una obra de teatro, y otro para cuarto, maratón de lectura. Ambos comprendieron una 
diversidad de actividades de aprendizaje. Aparejado al propósito de fomentar habilidades en el lenguaje, subyació el de estimular las competencias colaborativas y de expresión oral entre los niños, puesto que fueron dos debilidades que se observaron en el diagnóstico y durante las primeras semanas de la intervención.

Algunas de las actividades diseñadas tuvieron como objetivo incentivar la socialización e interesar al niño en LCM, pero sobre todo convencerlo de que él es protagonista en su proceso de aprendizaje. A los niños no se les trasmitieron estas palabras literalmente, pero el trabajo de colaboración desarrollado en el teatro LCM y coordinado por los "amigos" hizo sentir a los niños útiles y seguros de ellos mismos. También se fomentó la libertad de expresión y la toma de decisiones para un propósito de bien común, los mismos niños construyeron sus propios diálogos, diseñaron su vestuario y se apoyaron mutuamente. En el maratón de lectura se trabajó por equipos, y del mismo modo se destacó la importancia del trabajo conjunto para alcanzar las metas.

A pesar que ambas experiencias fueron enriquecedoras para todos los participantes, no estuvieron exentas de dificultades, sin embargo, eso también constituye un área de oportunidad para los universitarios, quienes se están formando precisamente para enfrentarse a un ambiente similar al vivido en este proyecto.

\section{Resultados}

\subsection{Dificultades y logros en la trasplantación de LCM al entorno mexicano}

Para el desarrollo de esta sección se realizaron entrevistas a 12 estudiantes que participaron en la intervención y a las tres profesoras a cargo de los grupos de la primaria. Fue un total de 13 entrevistas en las que vertieron puntos de vista sobre lo positivo y lo negativo de la experiencia. De lo analizado se presentan de manera sintética las principales ideas expresadas por los sujetos.

\subsubsection{La percepción de los estudiantes y profesoras}

En cuanto a las dificultades percibidas por los estudiantes, se agruparon en tres categorías: a) de organización y planificación, b) de capacitación, c) de trabajo en campo. La primera se relaciona con el diagnóstico escolar que no era lo suficientemente sólido para permitir desarrollar un plan de intervención completo y 
adaptado, aunado la falta de una implicación más decidida del equipo de trabajo de la Universidad. Respecto a la capacitación los estudiantes consideraron que fue insuficiente, y aunque su formación les ayudaba en las actividades didácticas, en otras dimensiones (como las habilidades de comunicación, pedagógicas o investigación) se sintieron en desventaja.

Con relación al trabajo de campo, los chicos se enfrentaron a una organización y normatividad rígidas que limitaban sus acciones, acompañado esto de un inicial desinterés por parte de los directivos y profesores de las escuelas. En el aula, se presentó el problema de la inexperiencia de los estudiantes para controlar algunas situaciones, como el uso del término "amigo", que por su connotación con una relación personal, evanesce la disciplina en los niños. Asimismo, existió la complejidad que planificar y diseñar actividades de aprendizaje innovadoras, así como redactar notas de campo, para lo cual los universitarios no se sintieron lo suficientemente preparados.

Sobre los logros, se destacan los tres más importantes: a) un impacto significativo en los valores, actitudes y autoestima de los niños que se expusieron al modelo, b) se estimularon sus habilidades comunicativas c) y los estudiantes universitarios que participaron en el proyecto obtuvieron experiencia y fortalecieron su práctica profesional. A pesar de que en los inicios se dificultó el manejo de la disciplina, el equipo de estudiantes logró establecer un sistema de valores entre los niños que fortaleció y facilitó el desarrollo de actividades y experiencias de aprendizaje. A medida que avanzaba el proyecto los niños tomaron mayor sentido de responsabilidad hacia LCM e incluso en clases normales.

La confianza que se generó entre los niños y los "amigos" permitió mayor apertura, los niños se expresaban con mayor libertad; hablar con ellos de sus problemas y conocer su ámbito afectivo emocional les permitió a los estudiantes universitarios entender el porqué de sus actitudes en el aula y conocer sus necesidades educativas individuales para buscar alternativas de apoyo.

"La verdad es que muchos niños introvertidos pudieron abrirse y expresarse mejor, que hasta la maestra se sorprendía, porque esos niños muy poco hablaban o participaban, en cambio con nosotros se volvieron muy activos, los niños se abrían a platicar de sus problemas y me di cuenta de cómo los niños traen en su cabecita tantas problemáticas que eso les impide avanzar" (Entrevistado 02). 
Niños que tenían pena o vergüenza para expresarse, lograron hacerlo mejor al final de la intervención. Se promovió el trabajo colaborativo y la equidad de género, niños a los que se les dificultaba trabajar en equipo al final lograron integrarse. Durante las primeras sesiones se negaban a conformar equipo con las niñas, pero se observó mayor disposición a medida que avanzaba LCM y el compañerismo caracterizó no solo las sesiones del programa sino también las de sus clases regulares. Se consiguió que los niños aprendieran a mostrar actitudes positivas en su proceso de aprendizaje; aprendieron a confiar en sí mismos, a no temer a equivocarse, a expresar sus dudas, a ayudar a los demás y a ser más participativos y respetuosos.

"Los niños aprendieron a confiar en personas, a entregarse como personas, a pesar de que algunos no se creían capaces de hacer algo por sí mismos, aprendieron a intentarlo" (Entrevistado 03).

Se dio un aprendizaje mutuo entre ambas partes. Los niños aprendieron a ser más independientes de manera responsable.

"Yo creo por ejemplo que Emiliano a pesar de ser un niño con TDH aprendió a escuchar, se apegaba mucho a una compañera, y creo que la confianza generada con ella le ayudó a disciplinarse sin necesidad de tener que reprimirlo o castigarlo, aprendió a colaborar" (Entrevistado 05).

Las habilidades comunicativas también se vieron estimuladas. Había niños tímidos, que en un principio hablaban entre dientes, en voz muy baja y sus compañeros los burlaban, pero con los valores que se promueven en La Clase Mágica de evitar las burlas y críticas, escuchar, apoyarse y motivarse, los niños superan muchas barreras y se expresan con más confianza “...lo logró y en el evento de fin de curso tomó el micrófono y compartió su experiencia y expuso su dibujo frente a toda la comunidad escolar" (Entrevistado 05). Leer diversos tipos de géneros literarios les fomentó el uso de nuevo vocabulario y los ayudó a mejorar su escritura. Estos avances se reflejan en los trabajos que realizaban en LCM como por ejemplo escribir a los reyes de España Isabel y Fernando, pero también en las encomiendas académicas que marca la maestra en sus clases obligatorias.

En el caso de los estudiantes, la mayoría no había trabajado con niños y LCM les 
permitió desarrollar esta capacidad, aprendieron técnicas de control grupal y disciplina, desarrollaron su creatividad planeando y diseñando actividades lúdicas. Este trabajo directo en el aula fortaleció sus habilidades docentes "...solucionar los problemas suscitados en el aula de clase con los niños es una competencia que muchos no teníamos y que nosotros fuimos desarrollando" (Entrevistado 06). Afirmaron que la ideología innovadora y sobre todo de carácter social de LCM les hizo cambiar su perspectiva en cuanto a las prácticas de enseñanza y aprendizaje tradicionales que se utilizan en las escuelas, implementaron una forma diferente (lúdica) de promover los aprendizajes en los niños.

Con respecto a las profesoras encargadas de los grupos de primaria que fueron espectadoras de la intervención, consideran que una limitante para el trabajo de los estudiantes es la "jerarquización" que se pierde entre los niños y los amigos. Piensan que estos últimos son incapaces de mantener el respeto cuando se establece una relación empática. Lo cierto es que los estudiantes universitarios manifiestan no poder controlar al inicio de la intervención la interacción con los niños.

Otra limitante de acuerdo a las docentes, es la falta de una planeación a largo plazo que los estudiantes no han sido capaces de ofrecer. Sin embargo, esto es factible de solventar. Lo importante es que entre los participantes se establezca una mística para la trasplantación.

\section{Reflexiones finales}

El modelo que hemos referido en la presente experiencia surgió como una alternativa de atender a grupos vulnerables, y se amplió para empoderarlos. Sus alcances son vastos así como sus beneficios. El proceso de trasplantación es complejo, sin embargo es clave para obtener resultados. En esta intervención se pueden destacar los siguientes aspectos:

En los niños participantes. Mostraron cambios positivos en su conducta colectiva, al inclinarse por el trabajo colaborativo. Se pudo observar comportamientos relacionados con valores como el respeto y la orientación al logro grupal, la convivencia y la integración. Para el proceso de conocimiento, de acuerdo a la teoría sociocultural (Vigotsky) son esenciales el uso de instrumentos socio culturales de dos tipos: herramientas y signos, como el lenguaje y escritura, que son producto de la interacción y de la evolución (Chaves, 2001). Son precisamente el uso de estrategias didácticas y 
artefactos (herramientas) y de interacciones (con los amigos) que se logró la apropiación de principios como el trabajo colectivo. El signo (Vigotsky, 2010) es un medio de vinculación social y llega a convertirse en un medio de acción sobre sí mismo.

En los estudiantes universitarios. Existe una latente ganancia al incursionar en contextos reales, lo que les permite este tipo de intervenciones. La inmersión en el sistema educativo nacional proporciona al estudiante herramientas para afrontar lo que tarde o temprano será su contexto laboral. Será un mediador, figura esencial en LCM, que ayude a la construcción del conocimiento a través de un proceso, lo cual representa uno de los principios de la Quinta Dimensión. Dicho proceso se basa en la colaboración entre actores que poseen conocimientos y experiencias distintas (Cole, 2006) y se encuentran en la Zona de Desarrollo Próximo bajo determinadas condiciones; es ahí donde el aprendiz (el niño) mediante la guía del experto (el universitario) adquiere autonomía en nuevas tareas, tal como sucedió en la intervención desarrollada.

Lo más sorprendente en esta propuesta teórica, es que otro de sus principios se refiere a los roles de experto y aprendiz, los cuales son flexibles, de modo que un mismo sujeto puede transitar entre uno y otro según los objetivos y actividades concretas. De ahí que los universitarios no solamente son la guía, sino que aprenden al mismo tiempo en el contexto, al poner en práctica los conocimientos teóricos aprendidos en su formación profesional.

En la escuela. Para la primaria, recibir jóvenes estudiantes que interactúen con los niños es refrescante. Permite que haya pautas de descanso y acomodo en la rutina diaria. El reforzamiento de los aprendizajes a través del juego representa una manera de mejorar el logro educativo. Para las profesoras representa una oportunidad de reorganizar sus actividades.

Para la universidad. Es un modo de mantener la interacción con el entorno educativo. Para la UJAT la responsabilidad social representa uno de los ejes transversales, y este tipo de estrategias son la oportunidad de ejercerla, y de realizar aportaciones en el sistema educativo de nivel básico. En este sentido el Plan de Desarrollo Institucional 2016 -2020 señala que las Divisiones Académicas están comprometidas con las condiciones de vida de las comunidades ( $p .125)$ para lo cual se demanda la participación amplia de la comunidad universitaria (p.149).

Sin embargo, pese a sus bondades, el modelo es complejo y tiene limitantes 
relacionadas sobre todo con el contexto, así como con la comprensión de los participantes sobre las implicaciones de su intervención. Es indispensable realizar un ejercicio de análisis sobre la trasplantación que facilite replicarlo. Sin embargo, parece que las ventajas sobrepasan sus debilidades, y vale la pena seguir diversificando sus aplicaciones.

\section{Bibliografía}

\section{PUBLICACIONES PERIÓDICAS}

Chaves, A. (2001). Implicaciones educativas de la teoría sociocultural de Vigotsky, Educación, 25, 59-65, Universidad de Costa Rica.

Crespo, I., Lalueza, J., Lamas, M., Prados, M. y Sánchez, S. (2014). El proyecto Shere Rom. Fundamentos de una comunidad de prácticas para la inclusión educativa de grupos culturales minoritarios y en riesgo de exclusión social. Psicología, Conocimiento y Sociedad, 4 (2), 138- 162. Recuperado de http://www.redalyc.org/articulo.oa?id=475847269007.

\section{LIBROS Y CAPÍTULOS DE LIBRO}

Alanís, I. (2014). Preparing aspirantes: sinchronizing culture and digital media. En B. Bustos, O. Vásquez, \& E. Riojas, La Clase Magina, generating transword pedagogy (pp.101-116). Maryland: Lexingtong Books.

Bustos, B., Vázquez , O., \& Riojas, E. (2014). ¡Adelante, el mundo nuevo!Educationg the new generationof the 21st century. En B. Bustos, O. Váquez, \& E. Riojas, La Clase Magina, generating transword pedagogy (pp. 13 -16). Maryland: Lexingtong Books.

Claeys, L. \& Muñoz, H. (2014). Unearthing sacred knowledge: enlazandonos con la comunidad. En B. Bustos, O. Vásques, \& E. Riojas, La Clase Magica, generating transworld pedagogy. (pp.67- 80). Maryland: Lexingtong Books.

Cole, M. (2006). The Fifth Dimension: an after-school program built on diversity. New York: Russell Sage Foundation.

Ek, L., García, A. \& Garza, A. (2014). Latino children:constructing identities, voices, linguistic and cultural understandings. En B. Bustos, O. Vásquez, \& E. Riojas, La Clase Magina, generating transword pedagogy. (pp.129 -142). Maryland: Lexingtong Books. 
Gunderman, H. (2008). El método de los estudios de caso. En M. Tarrés, Observar, escuchar y comprender, sobre la tradición cualitativa en la investigación social. (pp.249-284). México, México: El Colegio de México.

García, J., González, M. \& Ballesteros , B. (2001). Introducción a la Investigación en Educación. Madrid: UNED.

López, P. \& Valenzuela, A. (2014). Inserting La Clase magica into State-level policy discourses. En B. Bustos, O. Vásquez, \& E. Riojas, La Clase Magina, generating transword pedagogy. (pp.51-66). Maryland: Lexingtong Books.

Macías-Gómez, B. \& Vázquez, O. (2014). La Clase Magica goes international: adopting the new sociocultural context. En B. Bustos, O. Vásquez \& E. Rioja, La Clase Magina, generating transword pedagogy (pp.193-208). Maryland: Lexingtong Books.

Martínez, M. (2014). La Clase Magica, creating oporttunities for transformative learning. En B. Bustos, O. Vásquez, \& E. Riojas, La Clase Magica, generating transword pedagogy. (pp.33-50). Maryland: Lexingtong Books.

Martínez, M. (2012). Comunicación para el desarrollo y la inclusión social de minorías. La clase mágica: un modelo de intervención para el cambio social. Tesis Doctoral.

Martínez, P. (2006). El método de estudio de caso, estrategia metodológica de la investigación. Pensamiento y Gestión (20), 165-193.

Plan de Desarrollo Institucional 2016-2020 Universidad Juárez Autónoma de Tabasco.

Programa Sectorial de Educación 2013- 2018.

Rogoff, B. (1993). Aprendices del pensamiento el desarrollo cognitivo en el contexto social. Barcelona: Paidos.

Sánchez, P., Yuen, T., Shonle, M., De Hoyos, T., Santillán , L., \& García, A. (2014).

Digitizing El Laberinto. En B. Bustos, O. Vásquez, \& E. Riojas, La Clase Magina, generating transword pedagogy (pp.81-100). Maryland: Lexingtong Books.

Stake, R. (2007). Investigación con estudio de casos (Cuarta ed.). Madrid: Morata.

Vásquez, O. (2003). La Clase Mágica. Mahwah, New Jersey: Lawrence Erblsum Associated Publishers.

Vásquez, O. (2003). La Clase Mágica: Imagining optimal possibilities in a bilingual community of learners. Mahwah, New Jersey: Lawrence Erlbaum associates

Vigotsky, L. (2010) Pensamiento y Lenguaje, Madrid: Paidós. 
Willey, C., López, C., Torres, Z. \& Licon, L. (2014). Chanzas: the probability of changing the ecology of mathematics activity. En B. Bustos, O. Vásquez, \& E. Riojas, La Clase Magina, generating transword pedagogy (pp.159-176). Maryland: Lexingtong Books.

\section{MEDIOS ELECTRÓNICOS}

Herrera, S. (2005). Situación del ombudsman en el mundo. Revista de Comunicación, 4, 17-37 (www.saladeprensa.org/art-586.htm) (01-05-2008). (Se invita hacerlo acorde con el Manual de estilo de publicaciones (2002, 2ª edición en español) de la «American Psychological Association»).

Santos, L. (2016) ¿Cómo sostener el sitio? Documento presentado durante el Taller: ¿Cómo sostener La Clase Mágica en Tabasco? Impartido al equipo LCM en Tabasco. Villahermosa, Tabasco, México.

\section{REFERENCIA BIBLIOGRÁFICA}

García Martínez, V., Aquino Zúñiga, S. P. y López Laines, J. C. (2017). Experiencia en la trasplantación de la Clase Mágica al Entorno Mexicano. Aula de Encuentro, 19 (2), 76-96. doi: https://dx.doi.org/10.17561/ae.v19i2.4

Verónica García Martínez es Profesora investigadora de la División Académica de Educación y Artes de la Universidad Juárez Autónoma de Tabasco

Correo-e: veronica.garcia@ujat.mx

Silvia Patricia Aquino Zúñiga es Profesora investigadora de la División Académica de Educación y Artes de la Universidad Juárez Autónoma de Tabasco Correo-e: saquinozuniga@gmail.com 
Jessica del Carmen López Laines es

Egresada de la División Académica de

Educación y Artes de la

Universidad Juárez Autónoma de Tabasco

Correo-e: jessica.28junio@gmail.com

¿ Se decidió que fueran esos grados para poder continuar con la intervención longitudinalmente como lo establece el modelo original, y poder dar seguimiento al impacto del mismo entre los beneficiarios.

"El modelo de LCM posee ciertos "artefactos" que son distintivos de la propuesta, como El Laberinto, Las Rutas de Aprendizaje, Tarjetas de Tarea o El Maga, que constituyen elementos didácticos adaptables a un proyecto. 